\title{
Differential expression of plasma miR-146a in sepsis patients compared with non-sepsis-SIRS patients
}

\author{
LINA WANG ${ }^{1,2 *}$, HUA-CHENG WANG ${ }^{3 *}$, CHA CHEN $^{1}$, JIANMING ZENG ${ }^{1}$, \\ QIAN WANG ${ }^{2}$, LEI ZHENG ${ }^{2}$ and HUAN-DU YU ${ }^{4}$ \\ ${ }^{1}$ Department of Laboratory Medicine, Guangdong Provincial Hospital of Traditional Chinese Medicine, Guangzhou 510006; \\ ${ }^{2}$ Department of Laboratory Medicine, Nanfang Hospital, Southern Medical University, Guangzhou 510515; \\ ${ }^{3}$ Department of Laboratory Medicine, Guangzhou Brain Hospital, Guangzhou 510370; \\ ${ }^{4}$ Department of Laboratory Medicine, Kingmed Diagnostics, Guangzhou 510006, P.R. China
}

Received November 6, 2012; Accepted January 21, 2013

DOI: 10.3892/etm.2013.937

\begin{abstract}
Sepsis is a subtype of systemic inflammatory response syndrome (SIRS), which is characterized by infection. Circulating microRNAs (miRNAs), including miR-150, miR-146a and miR-223, are potential biomarkers of sepsis. In this study, we demonstrated that measuring the relative expression of miR-146a/U6 in plasma, using the $2^{-\Delta \Delta \mathrm{Ct}}$ method, provides a method for differentiating between sepsis and non-sepsis-SIRS. We observed a significant increase in miR146a expression in the initial cohort of 6 non-sepsis-SIRS patients compared to the 4 sepsis patients $(\mathrm{P}=0.01)$ and in the second cohort of 8 non-sepsis-SIRS patients compared to the 10 sepsis patients $(\mathrm{P}=0.027)$. Additionally, we identified that sodium citrate and ethylenediaminetetraacetic acid (EDTA) $\mathrm{K}_{2}$ may be used as anticoagulant reagents. Generation of a standard curve is not necessary in these diagnostic tests, unless the standard of normalization is carefully selected. Thus we provide more detailed guidance for the clinical use of circulating miRNA biomarkers.
\end{abstract}

\section{Introduction}

Sepsis is a life-threatening condition and the major cause of mortality among patients in intensive care units (ICUs).

Correspondence to: Dr Cha Chen, Department of Laboratory Medicine, Guangdong Provincial Hospital of Traditional Chinese Medicine, Guangzhou 510006, P.R. China

E-mail: chencha906@163.com

Dr Qian Wang, Department of Laboratory Medicine, Nanfang Hospital, Southern Medical University, Guangzhou 510515, P.R. China

E-mail:nflab@163.com

${ }^{*}$ Contributed equally

Key words: miR-146a, sepsis, diagnosis
The gold standard to distinguish sepsis from non-infectious diseases is blood microbiological culture analysis, which is more time-consuming than other potential biomarkers for early detection of sepsis, including acute phase proteins such as C-reactive protein (CRP) and procalcitonin (PCT). However, the lack of specifity of these more frequently used biomarkers impedes the significant requirement to identify novel biomarkers for early sepsis detection.

microRNAs (miRNAs) are a class of small (21-23 nucleotides), non-protein-coding RNAs that regulate the expression of target mRNAs post-transcriptionally. miRNAs are associated with a number of biological processes and the expression signatures of miRNAs serve as diagnostic and prognostic markers for various diseases. Numerous studies have established that the levels of miRNAs in serum or plasma are stable (4-7). After being normalized or quantified, circulating miRNAs yield high sensitivity and specificity by microarrays, next-generation sequencing or quantitative reverse transcription-polymerase chain reaction (RT-PCR) methods. miR-146a is one of the miRNAs that regulates the inflammatory response through a negative feedback nuclear factor

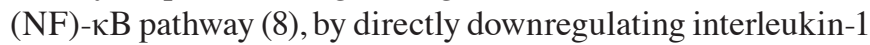
receptor-associated kinase 1 (IRAK1), tumor necrosis factor receptor-associated factor 6 (TRAF6) $(8,9)$ and IRAK2 (9). miR-146a may serve as a biomarker for rheumatoid arthritis (RA) (10) and sepsis (11). To investigate the clinical implication of miR-146a as a biomarker for sepsis diagnosis, we developed a qPCR-based precise quantification assay that reliably quantifies miR-146a (normalized to U6) from archived patient samples. We identified that plasma/serum miR-146a levels from sepsis patients were slightly decreased compared with non-sepsis-SIRS samples. However, different set points should be determined according to the control samples used during each experiment in clinical diagnostic practice.

\section{Materials and methods}

Participants. Blood samples were collected from 28 patients from Guangzhou Brain Hospital and Guangdong Provincial Hospital of Traditional Medicine, China. All patients provided 
informed consent. The study was approved by the ethics committee of Guangdong Provincial Hospital of Traditional Medicine, Guangzhou, China. Plasma samples in cohort 1 were collected and archived in April 2011 and plasma samples for cohort 2 were collected and archived between May and June 2011. All samples were collected when the first blood microbiological culture samples were collected. The results of blood microbiological culture determined whether patients had sepsis or non-sepsis-SIRS.

Plasma collection and RNA isolation. Both sodium citrate and ethylenediaminetetraacetic acid (EDTA) $\mathrm{K}_{2}$ anticoagulant samples were collected from the same patient at a particular time. Blood samples were centrifuged at $1,760 \mathrm{x}$ g for $10 \mathrm{~min}$ at room temperature. Plasma were obtained and mixed with TRIzol reagent (Dongsheng Biotech Co., Ltd., Guangzhou, China) at a ratio of $1: 2$ and stored at $-80^{\circ} \mathrm{C}$. Total RNA was extracted from serum according to the manufacturer's instructions.

Real-time quantitative RT-PCR. The RNA extracted from plasma were reverse transcribed as follows: a mixture consisting of $2 \mu \mathrm{l}$ RNA, $2 \mu \mathrm{l}$ stem-loop RT primer (RiboBio

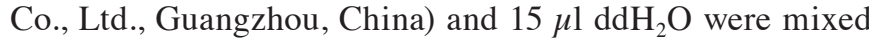
and incubated at $70^{\circ} \mathrm{C}$ for $10 \mathrm{~min}$ and then maintained at

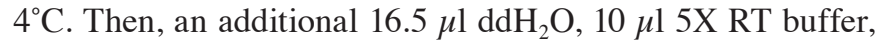
$2 \mu 12.5 \mathrm{nM}$ deoxynucleotide triphosphate (dNTP) and $2.5 \mu \mathrm{l}$ $200 \mathrm{U} / \mu 1$ PrimeScript RTase (Takara Bio Inc., Japan) were added, for a total volume of $50 \mu \mathrm{l}$. RT was performed on a Veriti ${ }^{\circledR}$ Thermal Cycler (Applied Biosystems, Carlsbad, CA, USA) at $30^{\circ} \mathrm{C}$ for $10 \mathrm{~min}, 42^{\circ} \mathrm{C}$ for $60 \mathrm{~min}, 70^{\circ} \mathrm{C}$ for $15 \mathrm{~min}$ and then held at $4^{\circ} \mathrm{C}$.

The EvaGreen PCR Master Mix (Bio-Rad, Hercules, CA, USA) was used to analyze the expression of miRNA. The U6 and miR-146a specific stem-loop amplification primers (RiboBio Co., Ltd.) were diluted to the working concentration. Then $9 \mu \mathrm{l}$ EvaGreen Master Mix was mixed with $4 \mu \mathrm{l}$ miRNA

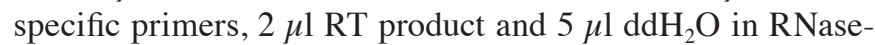
free strip-tubes. The qRT-PCR was then performed at $95^{\circ} \mathrm{C}$ for $20 \mathrm{sec}$, at $95^{\circ} \mathrm{C}$ for $10 \mathrm{sec}$ and at $60^{\circ} \mathrm{C}$ for $34 \mathrm{sec}$, with the last two steps repeated for 40 cycles. Then, the dissociation curve was generated. Data were analyzed with SDS software (Applied Biosystems), using the automatic Ct setting for assigning the threshold for $\mathrm{Ct}$ determination.

Standard curves were generated using synthetic miR-146a (RiboBio Co., Ltd), serially diluted and input into the RT reaction mixtures at the volume of $2 \mu \mathrm{l}$ per reaction.

Statistical analysis. Data were analyzed using the Mann-Whitney U test for the comparison of the different groups. The Wilcoxon matched pairs test was used to compare differences between the different anticoagulant groups. SPSS 13.0 software (SPSS Inc., Chicago, IL, USA) was used for all statistical analyses. Relative quantification using the $2^{-\Delta \Delta C t}$ method in the sepsis vs. non-sepsis-SIRS groups was carried out and fold changes were calculated for miR-146a.

\section{Results}

Normalization of miR-146a qRT-PCR data. Raw Ct values of U6 small nuclear RNA (snRNA) demonstrated no significant

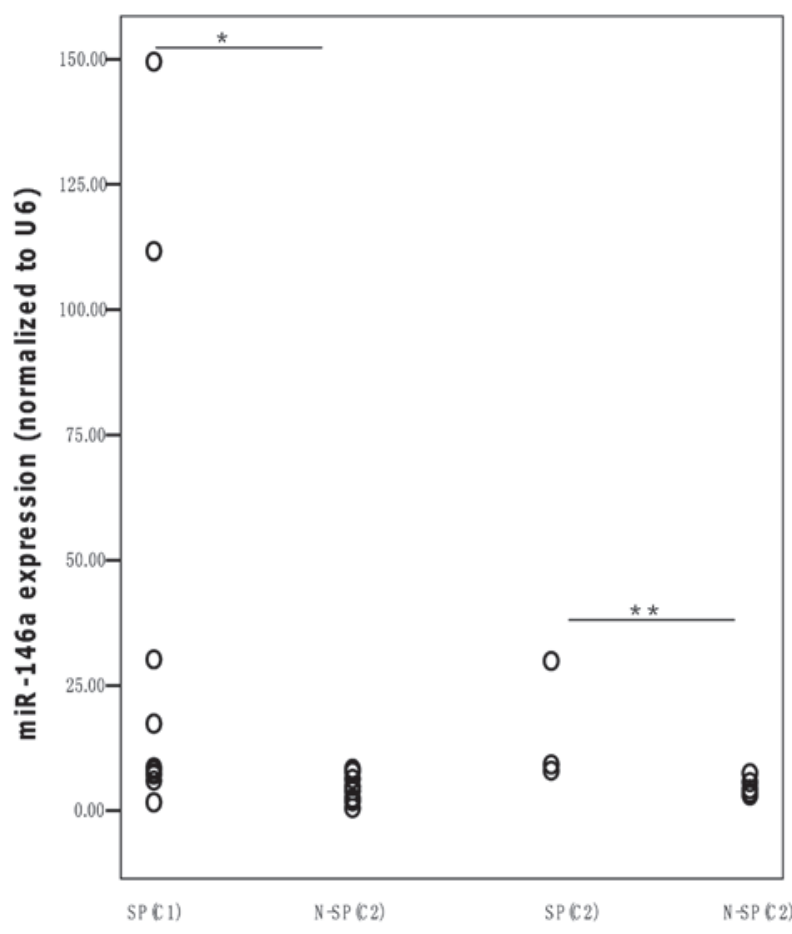

Figure 1. Comparison of miR-146a expression measures by real-time qRT-PCR. Data were normalized to U6 and were presented as $2^{[\mathrm{Ct}(\mathrm{miR}-146 \mathrm{a})-\mathrm{Ct}(\mathrm{U} 6)]}$. The levels of miR-146a were significantly reduced in the sepsis plasma samples. Triplicate assays were performed on each RNA sample. SP (C1), sepsis plasma samples in cohort $1(n=4)$; SP (C2), sepsis plasma samples in cohort $2(\mathrm{n}=6)$; N-SP $(\mathrm{C} 1)$, non-sepsis-SIRS plasma samples in cohort $1(\mathrm{n}=10)$; N-SP $(\mathrm{C} 2)$, non-sepsis-SIRS plasma samples in cohort $2(\mathrm{n}=8)$. Significant increases in miR-146a expression are indicated by ${ }^{*} \mathrm{P}=0027$ and ${ }^{* *} \mathrm{P}=0.01$. qRT-PCR, quantitative reverse transcription-polymerase chain reaction; SIRS, systemic inflammatory response syndrome.

differences between the sepsis and non-sepsis-SIRS groups $(\mathrm{P}=0.067$ in cohort 1 and $\mathrm{P}=0.122$ in cohort 2 ; Kruskal-Wallis test). Paired samples were collected in sodium citrate or ethylenediaminetetraacetic acid (EDTA) $\mathrm{K}_{2}$ anticoagulant tubes, respectively, from the same patient. No significant differences were detected in raw $\mathrm{Ct}$ values of U6 snRNA or miR-146a between the two anticoagulant groups.

In cohort 1, plasma miR-146a, normalized to U6 snRNA, demonstrated significant differences between the two groups, when normalized values were calculated as $2^{[\mathrm{Ct}(\mathrm{miR}-146 a)-\mathrm{Ct}(\mathrm{U} 6)]}$. Additionally, in cohort 2, plasma miR-146a in the sepsis group was significantly lower compared to that in the non-sepsis-SIRS group, when the same normalized values were used (Fig. 1).

Absolute quantification of miRNAs. The absolute expression levels of miR-146a were also calculated according to the standard curve of miR-146a (Fig. 2). No significant differences between the sepsis and non-sepsis-SIRS patients were identified in either cohort $(\mathrm{P}=0.762$ in cohort 1 and $\mathrm{P}=0.696$ in cohort 2). Additionally, no significant difference was observed when the absolute miR-146a levels were normalized to total RNA ( $\mathrm{P}=0.067$ in cohort 1 and $\mathrm{P}=0.274$ in cohort 2$)$.

Diagnostic value of miR-146a for sepsis. Receiver operating characteristics (ROC) curve analysis indicated that the area 


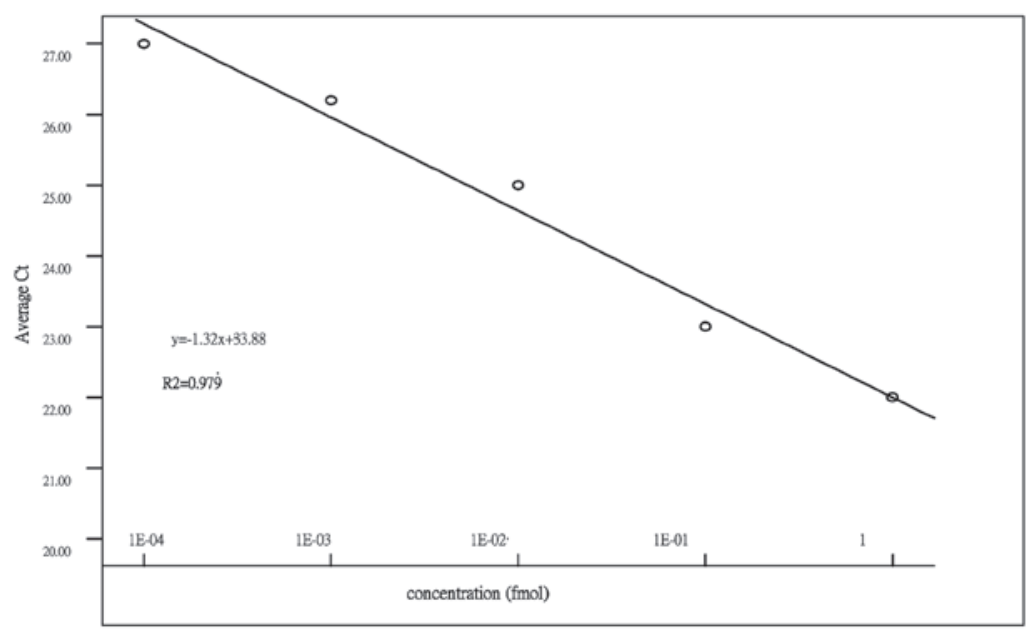

Figure 2. Standard curve for qRT-PCR assays generated for miR-146a using a dilution series of miR-146a mimics purchased from RiboBio Co., Ltd. qRT-PCR, quantitative reverse transcription-polymerase chain reaction.

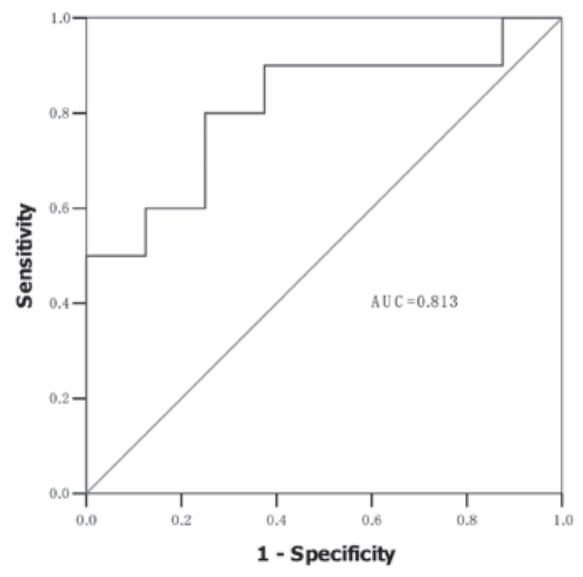

Figure 3. Receiver operating characteristics (ROC) curve analysis using serum miR-146a for discriminating sepsis patients from non-sepsisSIRS patients. The AUC (area under the ROC curve) was 0.813 (95\% CI, $0.608-1.017$ ) with a specificity of $87.5 \%$ and sensitivity of $60 \%$, respectively. SIRS, systemic inflammatory response syndrome.

under the curve (AUC) of miR-146a was 0.813 and at a cut-off point set at 7.97, miR-146a yielded a specificity of $87.5 \%$ and sensitivity of $60 \%$, respectively (cohort 2 ; Fig. 3).

\section{Discussion}

SIRS occurs when the body experiences an ongoing inflammatory response, which may arise from infectious or non-infectious triggers, including pancreatitis, trauma, drug fever and immunologic reactions. Sepsis is a potentially fatal condition characterized by a whole-body inflammatory state (12-14).

Sepsis may lead to multiple organ dysfunction syndrome (MODS) and mortality. A previous study demonstrated that for every hour delay in the appropriate antibiotic treatment of sepsis there is a $7 \%$ rise in mortality (15). The risk of mortality as a result of sepsis is strongly affected by the underlying disease. The 30-day survival rates for severe sepsis and septic shock patients are 65-80 and 40-60\%, respectively. The case-fatality rates are similar for culture-negative and culturepositive severe sepsis (16).

The majority of Gram-negative bacteria produce endotoxin, which causes fever and shock that are common symptoms during severe infections. Endotoxin binds to CD14 and then interacts with toll-like receptor 4 (TLR4). TLR2 is involved in the systemic response to Gram-positive bacteria infections (17). Studies are currently being performed to elucidate the mechanism of the response to these organisms.

Several circulating miRNAs, including miR-150, miR-182, miR-342-5p and miR-486 in peripheral blood mononuclear cells (PBMCs), miR-150 in plasma (18), and miR-146a and miR-223 in serum (11) are associated with sepsis. Nahid et al (19) identified that the miR-146a level was continuously elevated in THP-1 cells until $24 \mathrm{~h}$ after lipopolysaccharide (LPS) treatment. Taganov et al (8) identified that miR-146a/b, miR-132 and miR-155 are endotoxin-responsive genes and miR-146 is involved in the signaling cascade of TLR4/MyD88/IRAK1/TRAF6, which triggers the activa-

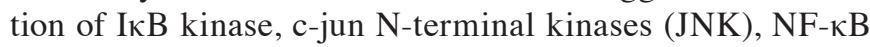
and activator protein (AP)-1, resulting in an upregulation of immune-responsive genes. Through a NF- $\mathrm{BB} / \mathrm{IRAK} 1 / \mathrm{TRAF6} /$ miR-146a negative feedback regulation loop, the activity of the inflammatory response is reduced.

There are several types of calibrators for absolute quantification of miRNAs by qRT-PCR, including plasmid (20), RNA fraction (21), cDNA samples (22) and synthetic target miRNA (23). For relative qRT-PCR, an appropriate reference gene must be selected. U6 snRNA was selected for the reference gene in this study since no difference in U6 snRNA levels has been detected in the serum/plasma of candidates with certain diseases and in control conditions (24-26), which was confirmed by our data. It was demonstrated that plasma miR-146a/U6 with the $2^{-\Delta \Delta C t}$ method yielded a similar AUC to serum miR-146a/cel-miR-39 with the $2^{-\Delta \Delta \mathrm{Ct}}$ method (11).

The role of miR-146a in the physiopathology of sepsis continues to be poorly understood. However, we demonstrated, using various sample types and normalized methods, that miR-146a may be used as a potential marker to differ- 
ence sepsis from non-sepsis-SIRS. It may be used in clinical practice as a complementary test for sepsis in patients where a diagnosis is not clear.

\section{References}

1. Simon L, Gauvin F, Amre DK, Saint-Louis P and Lacroix J: Serum procalcitonin and C-reactive protein levels as markers of bacterial infection: a systematic review and meta-analysis. Clin Infect Dis 39: 206-217, 2004

2. Tang BM, Eslick GD, Craig JC and McLean AS: Accuracy of procalcitonin for sepsis diagnosis in critically ill patients: systematic review and meta-analysis. Lancet Infect Dis 7: 210-217, 2007.

3. Young L: Gram-negative sepsis. In: Principles and Practice of Infectious Diseases. Mandell GL, Douglas RG, Bennett JE (eds). Churchill Livingstone, New York, pp611-636, 1990.

4. Chen X, Ba Y, Ma L, et al: Characterization of microRNAs in serum: a novel class of biomarkers for diagnosis of cancer and other diseases. Cell Res 18: 997-1006, 2008.

5. Mitchell PS, Parkin RK, Kroh EM, et al: Circulating microRNAs as stable blood-based markers for cancer detection. Proc Natl Acad Sci USA 105: 10513-10518, 2008.

6. McDonald JS, Milosevic D, Reddi HV, Grebe SK and Algeciras-Schimnich A: Analysis of circulating microRNA: preanalytical and analytical challenges. Clin Chem 57: 833-840, 2011.

7. Ho AS, Huang X, Cao H, et al: Circulating miR-210 as a novel hypoxia marker in pancreatic cancer. Transl Oncol 3: 109-113, 2010.

8. Taganov KD, Boldin MP, Chang KJ and Baltimore D: NF-kappaB dependent induction of microRNA miR-146, an inhibitor targeted to signaling proteins of innate immune responses. Proc Natl Acad Sci USA 103: 12481-12486, 2006.

9. Hou J, Wang P, Lin L, et al: MicroRNA-146a feedback inhibits RIGI-dependent type I IFN production in macrophages by targeting TRAF6, IRAK1 and IRAK2. J Immunol 183 2150-2158, 2009

10. Pauley KM, Satoh M, Chan AL, Bubb MR, Reeves WH and Chan EK: Upregulated miR-146a expression in peripheral blood mononuclear cells from rheumatoid arthritis patients. Arthritis Res Ther 10: R101, 2008.

11. Wang JF, Yu ML, Yu G, Bian JJ, Deng XM, Wan XJ and Zhu KM: Serum miR-146a and miR-223 as potential new biomarkers for sepsis. Biochem Biophys Res Commun 394: 184-188, 2010.

12. Bone RC, Balk RA, Cerra FC, et al: Definitions for sepsis and organ failure and guidelines for the use of innovative therapies in sepsis. Chest 101: 1644-1655, 1992.
13. Levy MM, Fink MP, Marshall JC, et al: 2001 SCCM/ESICM/ ACCP/ATS/SIS International Sepsis Definitions Conference. Crit Care Med 31: 1250-1256, 2003.

14. No authors listed: American College of Chest Physicians/Society of Critical Care Medicine Consensus Conference: definitions for sepsis and organ failure and guidelines for the use of innovative therapies in sepsis. Crit Care Med 20: 864-74, 1992.

15. Dellinger RP, Levy MM, Carlet JM, et al: Surviving sepsis sampaign: international guidelines for management of severe sepsis and septic shock: 2008. Crit Care Med 36: 296-327, 2008.

16. Shapiro NI, Wolfe RE, Moore RB, Smith E, Burdick E and Bates DW: Mortality in Emergency Department Sepsis (MEDS) score: a prospectively derived and validated clinical prediction rule. Critical Care Medicine 31: 670-675, 2003.

17. Takeuchi O, Hoshino K, Kawai T, et al: Differential roles of TLR2 and TLR4 in recognition of Gram-negative and Grampositive bacterial cell wall components. Immunity 11: 443-451, 1999.

18. Vasilescu C, Rossi S, Shimizu M, et al: MicroRNA fingerprints identify miR-150 as a plasma prognostic marker in patients with sepsis. PLoS One, 4, e7405, 2009.

19. Nahid MA, Pauley KM, Satoh M, et al: miR-146a is critical for endotoxin-induced tolerance, implication in innate immunity. J Biol Chem: 284, 34590-34599, 2009.

20. Formisano-Treziny C, de San Feliciano M and Gabert J: Development of plasmid calibrators for absolute quantification of miRNAs by using real-time qPCR. J Mol Diagn 14: 314-321, 2012.

21. Hromadnikova I, Kotlabova K, Doucha J, Dlouha K and Krofta L: Absolute and relative quantification of placenta-specific micrornas in maternal circulation with placental insufficiencyrelated complications. J Mol Diagn 14: 160-167, 2012.

22. $\mathrm{Pu}$ XX, Huang GL, Guo HQ, et al: Circulating miR-221 directly amplified from plasma is a potential diagnostic and prognostic marker of colorectal cancer and is correlated with p53 expression. J Gastroenterol Hepatol 25: 1674-1680, 2010.

23. Tomasetti M, Staffolani S, Nocchi L, et al: Clinical significance of circulating miR-126 quantification in malignant mesothelioma patients. Clin Biochem 45: 575-581, 2012.

24. Zhang Y, Jia Y, Zheng R, et al: Plasma microRNA-122 as a biomarker fro viral-, alcohol- and chemical-related hepatic diseases. Clin Chem 56: 1830-1838, 2010.

25. Starkey Lewis PJ, Dear J, Platt V, et al: Circulating microRNAs as potential markers of human drug-induced liver injury. Hepatology 54: 1767-1776, 2011.

26. Gui J, Tian Y, Wen X, et al: Serum microRNA characterization identifies miR-885-5p as a potential marker for detecting liver pathologies. Clin Sci (Lond) 120: 183-193, 2011. 